\title{
Jeux de marges
}

\section{Michel Lavigne}

\section{(2) OpenEdition}

Journals

Édition électronique

URL : http://journals.openedition.org/sdj/756

DOI : $10.4000 /$ sdj. 756

ISSN : 2269-2657

\section{Éditeur}

Laboratoire EXPERICE - Centre de Recherche Interuniversitaire Expérience Ressources Culturelles Education

\section{Référence électronique}

Michel Lavigne, « Jeux de marges », Sciences du jeu [En ligne], 7 | 2017, mis en ligne le 19 février 2017, consulté le 28 mars 2021. URL : http://journals.openedition.org/sdj/756 ; DOI : https://doi.org/ $10.4000 /$ sdj.756

Ce document a été généré automatiquement le 28 mars 2021.

\section{(2) $(\Theta \Theta \Theta$}

La revue Sciences du jeu est mise à disposition selon les termes de la Licence Creative Commons Attribution - Pas d'Utilisation Commerciale - Pas de Modification 4.0 International. 


\title{
Jeux de marges
}

\author{
Michel Lavigne
}

1 La notion de marge suppose l'existence d'une zone périphérique qui sépare un objet de ce qui lui est extérieur. Alors que la frontière est une séparation nette, une ligne, une coupure, la marge est une aire, un espace qui peut être vierge comme la marge autour d'un texte, mais qui peut aussi recevoir des annotations, voir accueillir un débordement du contenu. La marge peut devenir zone habitable: on peut «vivre en marge de la société ». Certes, la situation n'est pas toujours enviable ni clairement définie mais, à la faveur de la marginalité, peuvent s'inventer de nouveaux accommodements, dans l'acceptation de contraintes minimales qui permettent de garder des liens avec le groupe de référence tout en se tenant à l'écart. Etre à la marge c'est résider dans un entre-deux dont la nature est souvent difficile à déterminer. Une idée de flou peut s'y associer, voire une perspective de basculement potentiel d'un côté ou de l'autre.

2 Evoquer la notion de marge à propos du jeu suppose donc que l'on considère qu'il peut $\mathrm{y}$ avoir une zone intermédiaire entre ce qui relève du jeu et ce qui n'en relève pas. Cela conduit à porter son attention sur un territoire trouble et ne facilite pas l'appréhension du concept de jeu qui, dès lors, perd la netteté des contours que divers auteurs ont tenté de délimiter (Huizinga, 1988 [1951], Caillois, 1992 [1958]). Il est possible que cette zone de flou déborde sur le jeu lui-même rendant alors impossible sa délimitation. On peut aussi en espérer que puissent naître de nouvelles configurations.

3 Une seconde acception du terme marge peut retenir notre attention. La marge peut être un « espace dont on peut disposer entre des limites qui sont imposées » ou encore un «espace dans lequel peut s'exercer quelque chose ${ }^{1}$, tel que la "marge de manœuvre ». Dans ce cas il ne s'agit plus de l'aire périphérique d'un objet de référence, mais de celle au sein de laquelle, entre des bordures définies, peut s'exercer une certaine liberté de mouvement, une latitude. Ce type de marge peut aussi exprimer la situation du jeu, voire l'attitude du joueur.

4 C'est donc en tenant compte de cette polysémie que le jeu et la marge peuvent être confrontés. A cette complexité doit être ajoutée celle même de la notion de jeu. En effet, nous employons le terme jeu pour caractériser de multiples objets et situations 
disparates. Ludwig Wittgenstein (2014 [1953]) choisit d'ailleurs le terme jeu pour illustrer les difficultés du langage, constitué de concepts flous et impossibles à délimiter : nous savons quand et comment employer le mot mais nous ne savons pas le définir. Selon le philosophe, ce que nous qualifions de jeu peut constituer une famille, rassemblant des objets qui présentent des ressemblances, mais certainement pas une classe dont tous les membres partageraient des propriétés communes.

C'est dans le cadre de cette complexité, et en nous gardant de positions trop arrêtées, que notre réflexion s'attachera ici à envisager quelques configurations théoriques entre jeu et marge. Nous laissons aux auteurs de ce dossier le soin de développer ces articulations au sein de situations concrètes, qu'il s'agisse de jeu traditionnel ou de jeu numérique, pour de multiples jeux de marges.

\section{La frontière contre la marge}

Considérer qu'il peut exister une "marge du jeu » ne relève pas de l'évidence et se heurte à une conception traditionnelle bien ancrée en Occident. Le judéo-christianisme a tenu à distance le jeu, le reléguant dans le monde de l'enfance et de la frivolité, voire du péché. Si Thomas d'Aquin ne condamne pas le jeu, il recommande de le pratiquer avec la plus grande mesure et de le réserver au délassement (Duflo, 1997, p. 21). S'enracine ainsi une nette séparation, une frontière entre le monde du jeu et le reste des activités de la vie.

7 A l'école, le temps du jeu est celui de la récréation qui contraste avec le temps de la classe pendant lequel on ne joue pas. Pour les adultes le temps du jeu est celui du loisir, de la détente, mérité par les efforts dépensés au travail. Celui qui s'adonne au jeu de façon continue, oubliant les obligations «sérieuses" de la vie, s'expose à la condamnation morale. Une marge, un entre-deux, moitié jeu, moitié travail semble inconcevable : ou on joue ou on travaille.

Les conceptions du jeu développées par Joan Huizinga ou Roger Caillois sont héritières de cette tradition. Elles s'attachent à délimiter une frontière précise et étanche autour des activités considérées comme des jeux. Pour Huizinga (1988 [1951], p. 19) le jeu est "qualité déterminée d'action qui se distingue de la vie "courante" ". Selon l'historien, une des caractéristiques fondamentales du jeu réside dans cette stricte séparation, celui-ci étant rigoureusement cantonné dans un monde clos. Le jeu se déroule à l'intérieur de limites temporelles: "Le jeu commence et, à un certain moment, est "fini" » (p. 26). Il se déroule aussi à l'intérieur de limites spatiales : «Tout jeu se déroule dans les contours de son domaine spatial, tracé d'avance, qu'il soit matériel ou imaginaire, fixé par la volonté ou commandé par l'évidence » (p. 27).

En cela, l'historien assimile le territoire du jeu à ceux réservés à toutes les pratiques sacralisées : «L'arène, la table de jeu, le cercle magique, le temple, la scène, l'écran, le tribunal, ce sont là tous, quant à la forme et à la fonction, des terrains de jeu, c'est-àdire des lieux consacrés, séparés, clôturés, sanctifiés, et régis à l'intérieur de leur sphère par des règles particulières. » (Huizinga, 1988, p 27) Dès lors, pas plus qu'il ne semble possible de répandre le rite sacré hors du temple, que d'introduire des actes profanes en son sein, le jeu ne saurait être troublé par la « vie courante » sans perdre ce qui fait de lui son essence. 
10 A la suite d'Huizinga, Roger Caillois circonscrit avec précision les caractères qui, selon lui, définissent le jeu: «une activité: $1^{\circ}$ libre; $2^{\circ}$ séparée; $3^{\circ}$ incertaine; $4^{\circ}$ improductive ; $5^{\circ}$ réglée ; $6^{\circ}$ fictive ». (Caillois, 1992 p. 101) Ces « qualités » font du jeu un monde délimité par une frontière qui ne saurait tolérer l'indécision. «Opposant du moins fortement le monde du jeu au monde de la réalité, soulignant que le jeu est essentiellement une activité à part, elles laissent prévoir que toute contamination avec la vie courante risque de corrompre et de ruiner sa nature même " (Caillois, 1992, p. 101).

11 Cette position le conduit à s'interroger au long d'un chapitre entier sur la « corruption des jeux », c'est-à-dire ce qui advient "quand la cloison rigoureuse qui sépare leurs règles idéales des lois diffuses et insidieuses de l'existence quotidienne, perd sa netteté nécessaire. » (p. 101-102) Alors qu'il a défini plus tôt les quatre pulsions fondamentales qui animent notre désir de jeu (agôn, alea, mimicry, ilinx), le sociologue s'interroge sur leur devenir lorsqu'elles ne sont plus canalisées par ses conventions mais contaminées par la réalité. Lorsque «la vie courante » reprend ses droits, le jeu est corrompu, il disparait et se mue en des formes dégradées : la compétition sombre dans la violence, le respect du hasard se perd dans la superstition, le simulacre devient aliénation mentale ou dédoublement de la personnalité, la recherche du vertige déchoit dans les addictions à l'alcool ou aux drogues. Il y aurait un caractère civilisateur du jeu qui, par ses règles, impose une distance d'avec le réel. Les règles du jeu seraient à la fois «libératrices et isolantes » (Caillois, 1992, p. 113).

Les conceptions portées par Huizinga ou Caillois ne laissent pas de place pour penser une marge du jeu, mais seulement une frontière. Elles ont l'avantage de permettre une délimitation précise du phénomène de jeu. Dans cette perspective, s'intéresser aux marges du jeu n'est pas s'intéresser à un entre-deux mais bien à un hors-jeu.

\section{L'extension des marges du jeu}

Si Huizinga et Caillois ont proposé des définitions du jeu que l'on peut qualifier d'essentialistes, car elles le considèrent comme un phénomène universel inhérent à la condition humaine, voire animale, des conceptions plus modernes proposent une approche relativiste. Pour Jacques Henriot, l'idée de jeu n'est pas au fondement de la culture comme l'avance Huizinga, mais au contraire elle est une production culturelle qui, comme toute production culturelle, est liée à un contexte sociohistorique particulier. «Ce que l'on entend aujourd'hui par jeu, dans la société qui est la mienne, avait peut-être un contenu différent dans cette même société au cours des siècles passés, a peut-être une autre signification dans des groupes sociaux différents à l'époque où je vis et sera peut-être incompris des siècles futurs » (Henriot, 1989, p. 15).

De ce fait, l'idée de la séparation d'un jeu aux frontières étanche ne serait pas consubstantielle du concept de jeu, mais plutôt le produit de civilisations marquées par le sacré qui différencient jeu et vie courante de la même manière qu'elles opposent sacré et profane. Alors que le religieux s'efface dans les sociétés contemporaines, les stricts cloisonnements spatiaux et temporels établis par les sociétés traditionnelles perdent leur légitimité : « le jeu n'est plus limité [...] à des périodes strictement définies et strictement découpées dans la durée de l'existence collective. [...] Un nivellement se produit, qui constitue l'une des causes de la relative dilution et de l'instabilité de la 
notion de Fête [...] qui semble avoir perdu l'une de ses raisons d'être majeure: l'exercice de la différence » (Henriot, 1989, p. 30).

Plus loin, le philosophe ajoute : «s'il continue d'y avoir une opposition marquée entre les deux idées de Jeu et de Travail, cette opposition n'est plus aussi nettement tranchée, ne paraît plus aussi radicalement insurmontable qu'elle pouvait l'être au siècle dernier » (p. 58). Le jeu peut alors imprégner tous les actes de la vie sociale, de même que les actes de jeu ne se contentent plus d'être des parenthèses divertissantes. Avec les pédagogies nouvelles, le jeu entre dans la salle de classe et cherche sa place dans les méthodes éducatives. Dans le monde du travail, les managers ou les formateurs peuvent avoir recours à des jeux de rôle. Pour autant, de quel jeu s'agit-il ? A l'évidence le jeu évoqué n'est plus strictement distractif, il est dénaturé par des finalités extrinsèques.

Dans le même temps, l'idée de jeu s'immisce dans des situations qui n'ont rien de récréatif ni de futile. Henriot note cette extension de l'emploi du terme «jeu » au-delà des frontières du strictement ludique. Utilisé par les mathématiciens (la théorie des jeux), il en vient à caractériser des situations bien éloignées de l'amusement: "On admet aujourd'hui [...] qu'il puisse y avoir du jeu dans le "sérieux", du "sérieux" dans le jeu» (Henriot, 1989, p. 32). Le philosophe note que l'on parle aujourd'hui de «jeu politique ", de «jeu social» ou de «jeu du marché». La notion de jeu peut alors désigner toute sorte de situations présentant une certaine incertitude et faisant appel à des décisions stratégiques.

Cette extension du jeu qui multiplie les opportunités de situations imprécises et marginales serait le témoin d'évolutions sociétales profondes accordant une place croissante aux loisirs. Ce phénomène a été souligné par Alain Cotta (1980) dans La société ludique qui constate que la vie est envahie par le jeu, que ce soit avec le sport ou les jeux de hasard, alors que la menace d'ennui gagne une humanité qui n'est plus épuisée par la fatigue physique. Ce mouvement semble se renforcer dans l'époque contemporaine et la diffusion des jeux vidéo lui offre des impulsions nouvelles.

Le numérique donne lieu à de multiples débordements du jeu dans les activités du quotidien tout autant que du quotidien dans le jeu. Avec les jeux en ligne massivement multi-joueurs (MMORPG), les mondes persistants brouillent les anciennes frontières " entre le futile et le sérieux, la fiction et la réalité, l'intérieur et l'extérieur du jeu » (Schmoll, 2010, p. 30). Patrick Schmoll constate « la contamination de la réalité par le jeu » (p. 36) avec des « jeux sans fin». Les jeux pervasifs, qui superposent un modèle dit virtuel à la perception naturelle, rendent le réel jouable; les "serious games ${ }^{2}$ introduisent le jeu dans le monde de la formation ou de la prévention; le marketing prétend transformer le consommateur en joueur avec la gamification.

19 Les phénomènes de déplacements et d'extensions ludiques sont étudiés par Sébastien Genvo sous le terme de ludicisation (2013). Ce qui hier n'était pas considéré comme jeu peut le devenir, de la même façon que certains jeux peuvent quitter la sphère ludique, ce qui semble plus rare. De ce fait, la ludologie doit être attentive aux marges du jeu au sein desquelles se préparent les mouvements et futures transformations dans ce que nous considérons comme relevant du jeu. «En somme, il nous semble nécessaire de ne pas déduire les caractéristiques du jouable en nous fondant uniquement sur des objets identifiés comme des jeux, car cela risque d'en fixer les caractéristiques en fonction de ce qui est communément reconnu comme appartenant à la sphère ludique, alors que notre objectif est justement de pouvoir analyser ce qui se situe aussi à la marge des 
normes ludiques et les processus de transformation de ces normes» (Genvo, 2013, p. 66).

Les marges du jeu seraient alors ces espaces mouvants qui témoignent des transformations de notre perception du monde. Difficiles à appréhender, il faut en saisir la mobilité et l'instabilité pour comprendre comment se constitue notre idée du jeu et du non jeu en fonction de multiples influences contextuelles, à la fois d'ordre social et psychologique.

\section{Jouer dans la marge}

21 Si notre attention s'est portée jusqu'ici aux marges du jeu conçues comme éléments périphériques à l'activité ludique, le jeu lui-même peut être appréhendé comme une marge au sens de l'espace au sein duquel peut s'exercer une liberté dans le cadre des limites fixées par des règles. Pour Colas Duflo, « Le jeu est l'invention d'une liberté dans et par une légalité ». Le philosophe ajoute: «Dans tout jeu, le joueur possède une liberté de choix, de mouvement ou de décision.» (Duflo, 1997, p. 57). De ce point de vue les jeux ne sont pas tous égaux : certains sont pauvres, comme les jeux de hasard ou d'argent dans lesquels la seule possibilité de décision concerne le montant de la mise. D'autres au contraire, comme les jeux de rôles, offrent une grande liberté ludique qui requiert le développement de l'inventivité de la part du joueur. C'est là que réside, selon Duflo, un des plus grands intérêts des jeux: «C'est d'ailleurs de cette marge de liberté que naît une part de cette incertitude qui ne dépend point du hasard dans les jeux. » (p. 58)

Cette approche du jeu, en tant que marge de manœuvre, explique les évolutions de l'emploi du terme dans des situations non ludiques, comme par exemple avec l'expression de «jeu politique » que nous avons déjà citée. Ce type de jeu n'a rien de divertissant, ni de désintéressé, ni d'enfantin. Ce qui caractérise sa définition c'est la seule référence à ce possible mouvement dans un espace contraint.

En examinant la définition du mot jeu dans le Dictionnaire de la langue française de Littré, Jacques Henriot $(1989$, p. 86) constate trente et une acceptions du terme. Il focalise son attention sur une définition qui semble subalterne mais que le philosophe qualifie de "métaphore première" (p. 85): «aisance de mouvement, facilité à se mouvoir, en parlant des ouvrages d'art » (p. 87). On peut dans ce sens évoquer « le bois qui joue » ou l'intervalle permettant aux pièces mécaniques de s'emboîter entre elles sans se bloquer. Henriot caractérise, d'une façon plus générale, cette définition: "le mouvement relativement imprévisible, capricieux, de ce qui "joue" se produit dans des limites relativement déterminées, à l'intérieur d'un espace calculable [...] cet espace est un espace de jeu » (p. 90).

Si pour Caillois cette signification du jeu est secondaire, elle est essentielle pour Henriot. L'extension contemporaine de l'emploi du terme jeu semble lui donner raison, notamment avec le développement des simulations numériques ou des serious games: l'objectif n'est plus de se divertir mais de tester des possibilités d'action et leurs résultats. Il n'est pas surprenant que la "jouabilité » devienne une préoccupation centrale des concepteurs de jeux numériques, définissant les capacités d'actions offertes par un dispositif à un joueur et de nature à lui donner un sentiment de maitrise 
potentielle au sein d'une marge de manœuvre. Il faut pour jouer pouvoir agir sur une situation, disposer de possibilités de choix et de prise de décisions.

\section{Le « jouer » comme marge}

\section{Henriot (1989, p. 47) dissocie la «structure ludique» de «l'attitude ludique». Cette} dernière est le sentiment subjectif qui nous conduit à ressentir ou interpréter une situation comme un jeu. Si la jouabilité de la structure est une condition du jeu, cette potentialité d'action ne suffit pas pour produire du "jouer», encore faut-il que le joueur veuille agir " par jeu ». Il est difficile de se référer à l'idée de jeu lorsque l'on se trouve plongé au cœur de situations tragiques. Si l'on peut effectivement parler du «jeu politique », il paraîtrait inconvenant d'évoquer le « jeu du terrorisme » ou le « jeu de la guerre ». L'attitude ludique suppose un sentiment particulier de futilité ou de mise à distance comme le dénote Jacques Henriot. «L'idée de jeu est avant tout est avant tout affaire de point de vue. Elle suppose un survol, un relatif détachement, une sorte de légèreté mentale, au moins provisoire - qui n'a rien d'un retour à l'esprit d'enfance, mais qui amène à voir les choses d'une manière plus abstraite, plus théorique, moins immédiatement engagée » (Henriot, 1989, p. 53).

Nous sommes ici renvoyés à une appréciation relative, qui dépend d'un angle de vue ou d'un sentiment personnel qui nous fait vouloir «jouer le jeu». Cet état particulier est une situation fragile : « Le jouer menace constamment de se dissoudre, de faire place à quelque chose d'autre.» (Henriot, 1989, p. 149) Rapidement peut surgir l'ennui ou au contraire la rage de ne pas parvenir à réaliser les objectifs du jeu, ces deux limites conduisant au désengagement du joueur. "Ou bien, maintenant ses distances à l'égard d'un jeu dans lequel on refuse de se laisser prendre, on ne joue que distraitement, d'une manière détachée, désinvolte, sans trop y croire, en faisant à peu près n'importe quoi, en pensant à autre chose : alors on ne joue pas. Ou bien l'on joue "pour de bon", avec scrupule, passion, acharnement et le jeu, ainsi pris au sérieux, se mue en besogne en tâche absorbante, en obsession de tous les instants : alors on ne joue plus. Entre ces deux extrêmes, dont chacun trahit la prédominance excessive de l'une des composantes essentielles du jouer - improvisation et calcul, hasardement et réflexion - [...] où situer l'instant infinitésimal où l'on joue ?» (Henriot, 1989, p. 149)

Il semblerait alors que le jeu soit un point d'équilibre délicat, en permanence susceptible de se rompre. Mais l'acte de jeu, lorsqu'il perdure, prouve que l'équilibre est possible, peut-être dans un louvoiement entre de multiples menaces et de potentielles dérives périlleuses dans ses marges. Par sa théorie du flow, Mihaly Csikszentmihalyi (1990) illustre bien cette situation réussie qui maintiendrait le jeu vécu comme une « expérience optimale». Celle-ci se manifeste par un engagement du joueur dans une marge entre un état d'anxiété face à un obstacle insurmontable et l'ennui que peuvent produire des tâches répétitives ou sans intérêt.

Si l'attitude ludique se maintient dans une marge, le joueur lui-même se situe dans une marge par rapport à son jeu, celle-ci séparant sa conscience du jeu et la pratique ludique. « Le jouer dans son essence (c'est-à-dire : selon l'idée que l'on s'en fait) tient à la marge du jeu, à la distance qui se crée et se maintient entre le joueur et son jeu, entre ce qu'il est et ce qu'il fait, entre le sujet et le verbe de l'énonciation: "je joue" » (Henriot, 1989, p. 149). 
L'expérience de cette distance se trouve probablement à l'origine même de notre construction humaine et constitue un pivot de notre relation au monde. Selon Winnicott (2002 [1975]), le bébé pallie l'absence de sa mère et apprivoise le contact avec la réalité en inventant une aire imaginaire, une marge entre sa vie psychique intérieure et la réalité extérieure. Cet « espace potentiel » est un terrain d'expérimentation qui permet son développement personnel. Il est le territoire du jeu créatif ou playing. De ce fait, jeu et marge sont indissociablement liés et constituent un fondement essentiel de la condition humaine.

\section{Cinq articles pour des jeux de marges}

Les multiples recoupements entre jeu et marge offrent de fructueuses ouvertures à la pensée du jeu, que ce soit dans la réflexion sur l'extension sociale des manifestations ludiques, dans la considération des dispositifs internes de jeu, ou encore dans la relation entre le joueur et son jeu. La variété des thèmes abordés par les auteurs de ce numéro, tout comme celle de leurs approches et des terrains mobilisés, permet de donner un éclairage concret à ces diverses facettes des marges du jeu.

Les jeux vidéo sont devenus depuis une trentaine d'années une forme ludique dominante dans nos sociétés. Leur attractivité suscite la passion des publics, notamment des jeunes générations, proposant, au-delà de l'activité divertissante, de nouvelles références culturelles. Les situations et personnages de fiction qu'ils mettent en scène motivent une extension des pratiques vers des activités dérivées. Cette question est abordée par Pierre-Yves Hurel qui consacre ses recherches à la création de jeux vidéo en amateur à l'aide de logiciels spécialisés. Cette activité de création constitue-t-elle une marge du jeu ou un prolongement? Peut-on la considérer comme un nouveau jeu? Ou projette-t-elle son auteur hors-jeu?

En étudiant les parcours de créateurs de jeux vidéo en amateur, Hurel constate que ces pratiquants sont des joueurs passionnés depuis leur plus jeune âge, désirant prolonger leur plaisir dans l'invention de jeux imaginaires. Si la pratique du jeu en tant que telle cesse, l'appropriation d'un logiciel de création est elle-même vécue sur le mode du jeu, il ne s'agit plus de jouer au jeu vidéo mais de jouer $d u$ jeu vidéo. Alors que le passage vers une activité professionnelle semblerait possible, les pratiquants restent attachés à la gratuité de leurs actes. L'extension de la pratique ne conduit donc pas dans le hors $\mathrm{du}$ jeu, elle est renouvellement du jeu dans ses marges, car la figure de l'amateur reste dominante.

Nous avons évoqué la marge interne au jeu, induite par la constitution d'un dispositif particulier qui peut favoriser ou non l'attitude ludique. Deux auteurs de ce numéro se penchent sur cette question en examinant des objets ludiques. Boris Solinski s'intéresse aux livres-jeux qui posent la question de la frontière entre le jeu et la lecture. Entre ces deux activités que peuvent paraître opposées, quelles sont les configurations qui peuvent émerger? Selon Solinski, dans le cadre d'une approche structuraliste et fonctionnaliste, c'est par l'étude des potentialités de choix et des alternatives qu'elles offrent, que l'on peut représenter et apprécier le ludique, c'est dire la marge de manœuvre du joueur. A cette fin, après avoir fait une étude historique des livres-jeux et de leurs arbres de possibilités, il propose un panorama des divers types de choix. 

utilisent le monde réel dans un univers de jeu numérique. En prenant pour cas d'étude du JRA Alter Ego, ils fondent leur analyse sur l'observation du comportement des joueurs confrontés à de possibles confusions entre le monde du jeu et le monde réel. Ici apparaissent de nouvelles marges dans l'interprétation des situations relevant pour le joueur de cas d'indétermination entre ce qui relève effectivement du jeu et ce qui n'en fait pas partie. Ces indéterminations peuvent être créatrices ou destructrices au regard de la pratique du jeu. Si les indéterminations destructrices poussent vers le hors-jeu, les indéterminations créatrices apportent de nouvelles opportunités et enrichissements au jeu.

Nous avons enfin envisagé l'exercice de l'attitude ludique lui-même comme une marge, celle du joueur face au jeu, dans un mouvement qui ne relève pas de la structure du dispositif comme nous l'avons montré avec les deux articles précédents, mais de la disposition du joueur à le vivre ou non comme un jeu. C'est dans ce sens que Lydia Martin interroge la question de la perception de la marge entre jeu et travail dans l'utilisation d'un dispositif de type serious game. Que devient le jeu dans le cadre d'une pratique prescrite? L'interrogation de pratiquants fait ressortir la subjectivité de l'expérience vécue : selon l'histoire de vie de la personne la capacité à transférer une attitude ludique dans la vie professionnelle diffère. La même activité peut être perçue comme un jeu (capacité de duplicité) ou comme un exercice évaluatif culpabilisant.

De même, Adrien Czuser, en étudiant le monde particulier d'une communauté sadomasochiste (BDSM), envisage la capacité des personnes à vivre leurs relations sociales et sexuelles comme un jeu. Par l'attribution de rôles de dominants et de soumis, les activités BDSM pratiquées sont qualifiées de jeu par leurs adeptes. En recourant à la cadre-analyse de Goffman, l'auteur délimite le jeu BDSM avec ses marges interne et externe au sein desquelles jeu et non-jeu s'entremêlent. Le cadre primaire du jeu fournit une compétence interprétative de l'activité. Soumis à une forte variabilité d'appréciation par les acteurs, il requiert une capacité d'imagination créatrice qui permet de jouer dans et avec le jeu.

Ce dossier ne prétend pas faire le tour des rapports complexes entre jeu et marge. Il donne des ouvertures sur des territoires souvent flous et des équilibres instables ou délicats, que ce soit dans le domaine sociologique avec l'extension des pratiques de jeu, dans l'approche ludologique en questionnant les structures, ou encore en psychologie $\mathrm{du}$ fait des ambiguïtés de nos relations avec le jeu et le non-jeu. Il n'offre pas de réponses univoques, mais il invite à de multiples jeux de marges.

\section{BIBLIOGRAPHIE}

CAILLOIS R. (1992 [1958]), Les jeux et les hommes, Paris, Gallimard.

COTTA A. (1980), La société ludique - La vie envahie par le jeu, Paris, Bernard Grasset.

CSIKSZENTMIHALYI M. (1990), Vivre. La psychologie du bonheur, Paris, Robert Laffont. 
DUFLO C. (1997), Jouer et philosopher, Paris, Presses Universitaires de France.

GENVO S. (2013), Penser la formation et les évolutions du jeu sur support numérique, Mémoire pour l'habilitation à diriger des recherches en sciences de l'information et de la communication, Université de Lorraine.

HENRIOT J. (1989), Sous couleur de jouer, Paris, José Corti.

HUIZINGA J. (1988 [1951]), Homo ludens, Paris, Gallimard.

LAVIGNE Michel (2014), « Les faiblesses ludiques et pédagogiques des serious games », Colloque Ticemed 9, Toulon : https://www.academia.edu/6870543/

Les_faiblesses_ludiques_et_p\%C3\%A9dagogiques_des_serious_games

SCHMOLL P. (2010), « Jeux sans fin et société ludique », in S. Craipeau, S. Genvo \& B. Simonnot (eds), Les jeux vidéo au croisement du social, de l'art et de la culture, Metz, Questions de communication, Série Actes 8, p. p27-42.

WINNICOTT D. W. (2002 [1975]). Jeu et réalité, Paris, Gallimard.

WITTGENSTEIN L. (2014 [1953]), Recherches philosophiques, Paris, Gallimard.

\section{NOTES}

1. Définition du Trésor de la Langue Française Informatisé : http://atilf.atilf.fr/dendien/scripts/tlfiv5/advanced.exe?8;s=1470687765;

2. Nous conservons l'expression en langue anglaise afin d'indiquer que nous nous référons à une manifestation particulière du marketing des produits multimédias dans les années 2000. De la même façon que Jacques Henriot critique la croyance en l'existence ontologique du jeu chez Caillois, nous ne croyons pas en l'existence par essence du « jeu sérieux ». On pourra approfondir la question en consultant nos publications, notamment Lavigne (2014).

\section{RÉSUMÉS}

La confrontation des termes « jeu » et « marge » ouvre un large champ de réflexion car les deux concepts sont polysémiques et peuvent entretenir des rapports complexes. Si une acception traditionnelle du jeu le considère comme un champ clos, séparé par une nette frontière du nonjeu, l'évolution contemporaine voit une extension du ludique qui gagne vers ses marges et crée de nouvelles aires intermédiaires. La notion de marge peut aussi être convoquée pour évoquer le degré de liberté d'action laissé par un dispositif au joueur. On peut enfin s'intéresser à la marge constituée par la distance qui se crée entre le jeu et son joueur.

Confronting the words "game" and "margin" opens a wide field of thinking because the two concepts are polysemous and can maintain complex relationships. If a traditional understanding of gaming regards it as a closed field, separated by a definite frontier of non-game, contemporary evolution sees an expansion of the playful which gains towards its margins and creates new intermediate areas. The notion of margin can also be used to evoke the degree of freedom of 
action left by a device to the player. Finally, we can be interested in the margin constituted by the distance created between the game and its player.

\section{INDEX}

Mots-clés : jeu, marge, frontière, jeu vidéo, ludicisation, attitude ludique, expérience optimale, espace potentiel

Keywords : game, play, margin, border, video game, gamification, ludic attitude, optimal experience, potential space

\section{AUTEUR}

\section{MICHEL LAVIGNE}

Lara-Seppia, Université de Toulouse 